\title{
AGGREGATION IN THE PLANE AND LOEWNER'S EQUATION
}

\author{
L. CARLESON AND N. MAKAROV
}

\begin{abstract}
We study an aggregation process which can be viewed as a deterministic analogue of the DLA model in the plane, or as a regularized version of the Hele-Shaw problem. The process is defined in terms of the Loewner differential equation. Using complex analytic methods, we establish a Kestentype estimate for the growth of the cluster. We also indicate a real-variable approach based on a certain martingale structure in the phase space of the inverse Loewner chain.
\end{abstract}

\section{INTRODUCTION AND RESULTS}

Physical models such as the process of diffusion-limited aggregation (DLA) [23] and the Hele-Shaw problem [10], [19] make it natural to study general aggregation processes in the complex plane. The Loewner differential equation [14], which has been very successfully applied in classical function theory, seems to be an adequate tool to describe such processes. In this work we attempt to reach some understanding of the mechanism of Loewner's equation in the context of aggregation processes.

We will be considering a certain process defined in terms of Loewner's equation. This process can be viewed as a deterministic analogue of the DLA model, or as a regularized version of the Hele-Shaw equation. We establish a growth estimate similar to the one obtained by H. Kesten [11] for the lattice DLA. Two approaches are indicated. The first one is based on traditional methods of function theory and gives precisely the same bound as in Kesten's theorem. The second, real-variable approach is based entirely on the study of the inverse Loewner equation. Though we obtain a weaker estimate with the second approach, the method does not depend on the conformal structure and applies to more general evolution equations.

This section contains some preliminaries concerning the Loewner equation and aggregation processes, and also the statements of the results.

1.1. Loewner's equation. (See [1], [2], [17].) One can think of an aggregation process in the complex plane $\mathbb{C}$ as a growing family of connected compact sets $K_{t} \subset \mathbb{C}$ depending on some parameter $t$ ("time"), $t \in[0, T]$. The function

$$
c(t):=\operatorname{cap} K_{t}
$$

characterizes the speed of aggregation. (Recall that the logarithmic capacity of a connected set is comparable to the diameter.)

The second author is supported by N.S.F. Grant DMS-9800714. 
Let $\Omega_{t}$ denote the unbounded component of the set $\hat{\mathbb{C}} \backslash K_{t}$. The domains $\Omega_{t}$ are simply connected and satisfy the monotonicity condition

$$
s<t \Rightarrow \Omega_{t} \subset \Omega_{s}
$$

which is a setting for the Loewner differential equation. Namely, consider the family of conformal maps

$$
\varphi_{t}: \Delta \equiv\{|z|>1\} \rightarrow \Omega_{t}, \quad\left(\infty \mapsto \infty, \quad \varphi_{t}^{\prime}(\infty)>0\right),
$$

and assume that the functions

$$
c(t)=\varphi_{t}^{\prime}(\infty)
$$

are absolutely continuous. Then the function $t \mapsto \varphi(t, z) \equiv \varphi_{t}(z)$ is absolutely continuous for all $z \in \Delta$, and (1.1) implies the inequality

$$
\Re\left[\frac{\dot{\varphi}(t, z)}{z \varphi^{\prime}(t, z)}\right]>0
$$

( $\dot{\varphi}$ always means time derivative).

By Loewner's theory, the converse is also true. Let $A(t, z)$ be an analytic function (for each fixed $t$ ) satisfying

$$
\Re\left[\frac{A(t, z)}{z}\right]>0, \quad(z \in \Delta),
$$

and let $\left\{\mu_{t}\right\}$ be the corresponding family of Herglotz measures:

$$
A(t, z)=z \int_{\partial \Delta} \frac{z+\zeta}{z-\zeta} d \mu_{t}(\zeta) .
$$

If the function $t \mapsto\left\|\mu_{t}\right\|$ is locally integrable, then for any univalent function $\varphi_{0}$, the initial value problem

$$
\left\{\begin{array}{l}
\dot{\varphi}(t, z)=A(t, z) \varphi^{\prime}(t, z) \\
\left.\varphi\right|_{t=0}=\varphi_{0}
\end{array}\right.
$$

has a unique solution, and the solution is a Loewner chain, i.e. a family of univalent functions satisfying (1.1) and (1.2). We will call $\mu_{t}$ the growth measures of the Loewner chain. Note that

$$
\dot{c}(t)=\left\|\mu_{t}\right\| c(t) .
$$

It follows that there is a one-to-one correspondence between (locally integrable) families of positive measures $\left\{\mu_{t}\right\}$ on the unit circle and (absolutely continuous) Loewner chains starting with a given univalent function $\varphi_{0}$. This correspondence allows to describe aggregation processes in one dimensional terms.

In the following two examples, it is easy to interpret growth measures in terms of the aggregation.

(i) Suppose first that the measures $\mu_{t}$ are absolutely continuous:

$$
d \mu_{t}(\zeta)=\rho_{t}(\zeta)|d \zeta|, \quad(\zeta \in \partial \Delta)
$$

and suppose that $\Omega_{t}$ are Jordan domains with smooth boundaries. Then

$$
\rho_{t}(\zeta)=V_{n}(a)\left|\nabla G_{t}(a)\right|, \quad\left(a:=\varphi_{t}(\zeta)\right),
$$


where $V_{n}$ is the normal velocity of the boundary, and $G_{t}(\cdot)$ is the Green function of $\Omega_{t}$ with pole at infinity. Indeed, the interior normal of $\Omega_{t}$ is given by the unit vector

$$
n(a)=\frac{\zeta \varphi_{t}^{\prime}(\zeta)}{\left|\varphi_{t}^{\prime}(\zeta)\right|}
$$

Applying (1.3), we have

$$
\begin{aligned}
V_{n}(a) & =<\dot{\varphi}_{t}(\zeta), n(a)>=\frac{\Re\left[\dot{\varphi}_{t} \bar{\zeta} \bar{\varphi}_{t}^{\prime}\right]}{\left|\varphi_{t}^{\prime}\right|} \\
& =\left|\varphi_{t}^{\prime}(\zeta)\right| P(\zeta)=\left|\varphi_{t}^{\prime}(\zeta)\right| \rho_{t}(\zeta),
\end{aligned}
$$

where $P(\cdot)$ is the Poisson integral of $\mu_{t}$.

(ii) Consider now the case of pure point growth measures. The simplest situation is when $\mu_{t}$ is constant, say

$$
\mu_{t} \equiv \text { const } \delta_{\zeta}, \quad\left(t_{1} \leq t \leq t_{2}\right),
$$

for some point $\zeta \in \partial \Delta$. Then the map $\varphi_{t_{2}}$ is a composition

$$
\varphi_{t_{2}}=\varphi_{t_{1}} \circ h
$$

of $\varphi_{t_{1}}$ and a univalent function $h$ which maps $\Delta$ onto the domain

$$
\Delta \backslash\{r \zeta: 1 \leq r \leq 1+\varepsilon\} .
$$

The length $\varepsilon$ of the slit satisfies the equation

$$
\frac{(2+\varepsilon)^{2}}{4(1+\varepsilon)}=e^{\Delta \mu}, \quad \Delta \mu:=\int_{y_{1}}^{t_{2}}\left\|\mu_{t}\right\| d t
$$

in particular, we have

$$
\varepsilon^{2} \sim 4 \Delta \mu \quad \text { as } \quad \Delta \mu \rightarrow 0 .
$$

Thus the domain $\Omega_{t_{2}}$ is obtained from $\Omega_{t_{1}}$ by cutting the latter along a geodesic segment.

Classical Loewner chains correspond to the case $\mu_{t}=\delta_{\zeta(t)}$, where $\zeta(t)$ is a continuous function. If this function is sufficiently smooth, then the previous example gives an infinitesimal description of the aggregation; the interpretation becomes rather delicate for non-smooth functions, see [15].

The Loewner equation (1.3) is equivalent to a first order linear PDE in three real variables. According to the general theory, a first order PDE can be solved in terms of an ordinary differential equation - the equation of characteristics. The method of characteristics has the following form in the case of Loewner's equation. For $s \leq t$, we can define the functions

$$
h_{s, t}:=\varphi_{s}^{-1} \circ \varphi_{t},
$$

which are univalent and satisfy the inverse equation

$$
\partial_{s} h_{s, t}(z)=-A\left(s, h_{s, t}(z)\right) .
$$

Solutions $w=w(s)$ of the equation

$$
\partial_{s} w=-A(s, w)
$$


are called characteristics of the Loewner equation. It follows that if $w(\cdot)=w(\cdot ; t, z)$ denotes the solution of the initial value problem $w(t)=z$, then $w(s ; t, z)=h_{s, t}(z)$, and so the chain $\left\{\varphi_{t}\right\}$ can be expressed in terms of characteristics:

$$
\varphi_{t}(z)=\varphi_{0} \circ w(0 ; t, z) .
$$

1.2. Aggregation processes. An interesting class of processes is the following. Suppose that at any time $t$, the growth measure $\mu_{t}$ of a Loewner chain is determined by the domain $\Omega_{t}$, the state of the process. Since $\Omega_{t}$ is in turn determined by the family $\left\{\mu_{s}\right\}_{s<t}$, the growth measures are subject to some deterministic equation. There is also a stochastic version. The growth measures are random, but the probability law of $\mu_{t}$ depends on $\Omega_{t}$, in which case we have a measure-valued Markov process. Several important processes of this type are known from the literature. We mention two examples in order to give some motivation for the process to be defined later.

Let us first introduce the following notation. Fix a small number $\delta>0$. Given a conformal map $\varphi: \Delta \rightarrow \Omega,(\infty \mapsto \infty)$, we define a positive function $\varepsilon(\zeta) \equiv \varepsilon(\zeta ; \delta)$ on $\partial \Delta$ by the equation

$$
\varepsilon(\zeta)=\inf \{\varepsilon: \operatorname{dist}(\varphi(\zeta+\varepsilon \zeta), \partial \Omega)=\delta\} .
$$

Since for all $\zeta \in \partial \Delta$, we have

$$
\left|\varphi^{\prime}(r \zeta)\right|=o\left(\frac{1}{r-1}\right) \quad \text { as } \quad r \rightarrow 1,
$$

the function $\varepsilon(\zeta)$ is defined and is positive everywhere on the circle. If $\left\{\varphi_{t}\right\}$ is a Loewner chain, then we write $\varepsilon_{t}(\zeta)$ for the corresponding functions (1.8).

Example 1 (a version of the DLA model). Consider the arriving times $\tau_{j}$ of a Poisson process (with mean one) and, independently, consider a sequence of independent random variables $\zeta_{j}$ uniformly distributed on the unit circle. The growth measures are defined by the equation

$$
\int_{0}^{t} \mu_{s} d s=\sum_{\tau_{j} \leq t} \varepsilon_{\tau_{j}}^{2}\left(\zeta_{j}\right) \delta_{\zeta_{j}} .
$$

The equation means that at Poisson random time, we choose a random point on the boundary of the growing cluster with probability law given by harmonic measure at that moment, and then we add a segment of length $\asymp \delta$ of the corresponding geodesic, see (1.6).

The process (1.9) resembles the standard DLA model [23], see also [3], [22], and computer simulations produce similar pictures, see [16], [9]. Recall that in the lattice version of the DLA process, particles are released near infinity and perform symmetric random walk on $\mathbb{Z}^{2}$ until they reach a point of the growing cluster, where they stick to the cluster. The hitting probability of the random walk is the lattice analogue of harmonic measure.

Example 2 (Hele-Shaw equation without surface tension). The following is an example of a deterministic process, see, e.g. [19], [7], [8], [21]. Let us assume that the boundaries $\partial \Omega_{t}$ are smooth in some time interval (it is known that this assumption 
holds only if the initial domain has real analytic boundary) and require that the normal velocity of the boundary be equal to the gradient of the Green function:

$$
V_{n}=\left|\nabla G_{t}\right| \text {. }
$$

By (1.5), the latter means that the growth measures satisfy the equation

$$
d \mu_{t}(\zeta)=\left|\varphi_{t}^{\prime}(\zeta)\right|^{-2}|d \zeta|
$$

Definition. Let $\delta$ be a positive number. We say that a Loewner chain $\left\{\varphi_{t}\right\}$ is an $\operatorname{HS}(\delta)$-process if the growth measures satisfy the equation

$$
d \mu_{t}(\zeta)=\varepsilon_{t}^{2}(\zeta ; \delta)|d \zeta|
$$

One can interpret an $\operatorname{HS}(\delta)$-process as a deterministic version of the DLA model. Namely, consider the stochastic equation (1.9) of Example 1, and let $e$ be an arbitrary measurable subset of the unit circle. Then we have the following expression for the conditional expectation:

$$
E\left(\int_{t}^{t+\Delta t} \mu_{s}(e) d s \mid \Omega_{t}\right)=\Delta t \int_{e} \varepsilon_{t}^{2}(\zeta)|d \zeta|+o(\Delta t) \quad \text { as } \quad \Delta t \rightarrow 0 .
$$

Dividing by $\Delta t$ and letting $\Delta t \rightarrow 0$, we obtain (1.11).

On the other hand, $\mathrm{HS}(\delta)$-chains can be viewed as solutions to a regularized or "smoothed" Hele-Shaw problem (see Example 2). By the distortion theorem, we have

$$
\varepsilon \asymp \frac{\delta}{\left|\varphi_{t}^{\prime}(\zeta+\varepsilon \zeta)\right|}, \quad \varepsilon:=\varepsilon_{t}(\zeta ; \delta)
$$

and so if we make an appropriate change of the time variable in the equation (1.11), then (1.10) will be its formal limiting case as $\delta \rightarrow 0$. In contrast to the ill-posed Hele-Shaw problem, the equation (1.11) is well-posed. It should be mentioned that our regularization is different from the "viscosity" type regularization usually considered in hydrodynamics.

Speaking of aggregation processes, we can't help mentioning a spectacular recent work of Lawler, Schramm and Werner [13] on Brownian intersection exponents, in which a certain process $\mathrm{SLE}_{6}$ plays a key role. The Schramm-Loewner evolution process $\mathrm{SLE}_{\kappa}$ with parameter $\kappa>0$ is defined by the equation $\mu_{t}=\delta_{B(\kappa t)}$, where $B(t)$ is the standard Brownian motion on the circle $\mathbb{R} / \mathbb{Z}$ starting at a uniformrandom point, see [20].

1.3. A growth estimate. H. Kesten [11] established the following theorem for the lattice DLA model mentioned above:

There is a universal constant $C$ such that with probability one, the diameter $D_{N}$ of a DLA cluster with $N$ particles satisfies the inequality

$$
D_{N} \leq C N^{2 / 3}
$$

for all sufficiently large $N$. 
This result is a consequence of the estimate

$$
T \gtrsim D^{3 / 2}
$$

for the doubling time $T$. Here $D$ denotes the diameter of the cluster with $N$ particles, and $T$ is the minimal number such that the cluster with $N+T$ particles has diameter $2 D$. It is claimed that (1.12) is true with probability large enough so that one can apply the Borel-Cantelli lemma.

The following theorem is a deterministic counterpart of Kesten's theorem.

Theorem A. Let $\left\{\varphi_{t}\right\}$ be an $\mathrm{HS}(\delta)$-process for some positive $\delta<1$, and suppose that

$$
\left|\varphi_{0}^{\prime}(\infty)\right|=1, \quad\left|\varphi_{T}^{\prime}(\infty)\right|=10
$$

Then

$$
T \gtrsim \delta^{-3 / 2}
$$

Equations (1.13) mean that $T$ is essentially the doubling time for the logarithmic capacity of the cluster, and so the estimates (1.12) and (1.14) are quite similar.

On the other hand, it is not clear if one can extend Kesten's argument (see also the proof in [14]) to our deterministic situation. In addition to probability considerations and a Beurling-type estimate for harmonic measure, the proof of in [11] depends on a bound for the number of all possible non-selfintersecting lattice paths in a region of a given diameter.

In the case of an $\mathrm{HS}(\delta)$-process, the Beurling estimate has the form

$$
\varepsilon_{t}(\zeta ; \delta) \lesssim \sqrt{\delta}, \quad(0 \leq t \leq T)
$$

Since (1.13) implies

$$
\int_{0}^{T} d t \int_{\partial \Delta} \varepsilon_{t}^{2}(\zeta ; \delta)|d \zeta| \asymp 1,
$$

see (1.4), we immediately get $T \gtrsim 1 / \delta$. The following universal estimate for the integral means of $\varepsilon^{2}$ gives a better bound. Recall that the class $(\Sigma)$ consists of univalent functions $\varphi$ in $\Delta$ with normalization $\varphi(\infty)=\infty$ and $\varphi^{\prime}(\infty)=1$.

Proposition. There are positive absolute constants $c$ and $C$ such that if $\varphi \in(\Sigma)$ and $0<\delta<1$, then

$$
\int_{\partial \Delta} \varepsilon^{2}(\zeta ; \delta)|d \zeta| \leq C \delta^{1+c} .
$$

This proposition is a consequence of the following fact established in [5]. Denote $\Omega:=\varphi \Delta$ and let $\omega$ be the harmonic measure of $\Omega$ evaluated at infinity. Then the maximal number of disjoint discs of radius $\delta$ and harmonic measure $\geq \delta^{(1+c) / 2}$ does not exceed

$$
A \delta^{-\gamma(c)} \text { with } \gamma(c)=o(c) \quad \text { as } \quad c \rightarrow 0
$$

where the constant $A$ and the function $\gamma(c)$ do not depend on $\varphi \in(\Sigma)$. (In [6], it is shown that one can take $\gamma(c)=K c$ for some absolute constant $K \geq 2$.)

To derive (1.16) from (1.17), we cover the unit circle with disjoint dyadic intervals $l_{\nu}$ such that

$$
\max _{\zeta \in l_{\nu}} \varepsilon(\zeta) \asymp\left|l_{\nu}\right|,
$$


which is possible by a simple stopping time argument. It follows that

$$
\int_{\partial \Delta} \varepsilon^{2}(\zeta)|d \zeta| \lesssim \sum_{\nu}\left|l_{\nu}\right|^{3}
$$

Denote by $\zeta_{\nu}$ the center of the interval $l_{\nu}$, and let $a_{\nu}:=\varphi\left(\zeta_{\nu}+\left|l_{\nu}\right| \zeta_{\nu}\right)$. Then

$$
\operatorname{dist}\left(a_{\nu}, \partial \Omega\right) \asymp \delta
$$

by the distortion theorem, and

$$
\omega B\left(a_{\nu}, \lambda \delta\right) \gtrsim\left|l_{\nu}\right|
$$

for some absolute constant $\lambda>1$. The discs $B\left(a_{\nu}, \lambda \delta\right)$ are essentially disjoint because

$$
\left|a_{\nu}-a_{\nu^{\prime}}\right| \gtrsim \delta, \quad\left(\nu \neq \nu^{\prime}\right)
$$

by construction. Applying (1.17), we see that the number of intervals $l_{\nu}$ such that $\left|l_{\nu}\right|>\delta^{(1+c) / 2}$ is $\lesssim \delta^{-\gamma(c)}$, and so the sum $\sum\left|l_{\nu}\right|^{3}$ taken over such intervals is

$$
\lesssim \delta^{3 / 2-\gamma(c)} \leq \delta^{1+c},
$$

provided that $c$ is small enough to make $c+\gamma(c)<1 / 2$. Since $\sum\left|l_{\nu}\right|=1$, the corresponding sum taken over intervals satisfying $\left|l_{\nu}\right| \leq \delta^{(1+c) / 2}$ also does not exceed $\delta^{1+c}$ by Beurling's estimate.

It follows that for $\operatorname{HS}(\delta)$-processes, we have

$$
T \gtrsim \delta^{-(1+c)}
$$

with the same constant $c$ as in (1.16). However, we can not take $c=1 / 2$ in (1.16), which would immediately give the Kesten-type result. In fact, it was shown in [6] that if $q<3$, then there are functions $\varphi \in(\Sigma)$ satisfying

$$
\int_{\partial \Delta} \varepsilon^{q}(\zeta ; \delta)|d \zeta| \gtrsim \delta^{\gamma} \quad \text { with } \quad \gamma<\frac{1+q}{2},
$$

(according to Brennan's conjecture [4] the value $q=3$ is critical.) What Theorem A actually states is that an $\operatorname{HS}(\delta)$-process can not spend much time in configurations satisfying (1.18).

We prove Theorem A in Section 2. The main idea is to localize (by means of Poisson averages) the bound (1.16) near the points corresponding to the "tips" of the cluster. Non-local estimates of this type are well-known in the conformal mapping theory; they are related to the Muckenhaupt $\left(A_{\infty}\right)$ condition and characterize the degree of smoothness of the boundary, see [18], Chapter 7 .

It may be worth mentioning that the bound (1.14) is best possible, at least formally. It is easy to see that if an $\operatorname{HS}(\delta)$-chain starts, for example, with the function

then we have

$$
\varphi_{0}(z)=z+z^{-1}
$$

$$
\int_{\partial \Delta} \varepsilon_{t}^{2}(\zeta ; \delta)|d \zeta| \asymp \delta^{3 / 2}, \quad(0 \leq t \leq T),
$$

and therefore $T \asymp \delta^{-3 / 2}$ in this case. This fact is similar to the phenomenon of "finger" solutions in the Hele-Shaw problem, see [19]. We don't know if (1.14) is best possible for "generic" initial configurations. 
1.4. Loewner's method. The Loewner equation provides one of the most powerful methods in dealing with problems concerning functionals such as the coefficients of univalent functions. In connection with aggregation processes, we would like to understand how one can apply Loewner's method to quantities characterizing dimensional properties of conformal maps such as integrals $\int \varepsilon^{q}$, or integral means of the derivative.

Let us consider a Loewner chain of functions $\varphi_{t}$ defined in the upper halfplane $\{\Im z>0\}$, and assume that $\varphi_{0}=\mathrm{id}$. (The formulae are slightly simpler in this case.) Then the potential $A(t, z)$ in Loewner's equations (1.3) and (1.7) has the form

$$
A(t, z)=\int_{\mathbb{R}} \frac{d \mu_{t}(\xi)}{\xi-z}
$$

Differentiating the inverse equation (1.7) with respect to $z$, and then integrating over $[0, t]$, we have

$$
\log \left|\varphi^{\prime}(t, z)\right|=\int_{0}^{t} \Re\left[A^{\prime}(s, w(s))\right] d s
$$

where $w(s):=w(s ; t, z)$ is the corresponding characteristic. Similarly, we find

$$
\log \frac{\Im[\varphi(t, z)]}{\Im z}=\int_{0}^{t} M(s, w(s)) d s
$$

where

$$
M(s, x+i y)=\int \frac{d \mu_{s}(\xi)}{(\xi-x)^{2}+y^{2}} .
$$

Note that the sum $K:=\Re\left[A^{\prime}\right]+M$ of the kernels in (1.19) and (1.20) is positive:

$$
K(s, x+i y)=\int \frac{2(\xi-x)^{2}}{\left[(\xi-x)^{2}+y^{2}\right]^{2}} d \mu_{s}(\xi) .
$$

For a fixed $\delta>0$, let $\rho=\rho(t, x)$ be such that $\operatorname{dist}\left(\varphi_{t}(x+i \rho), \partial \Omega_{t}\right) \asymp \delta$, i.e.

$$
\rho\left|\varphi_{t}^{\prime}(x+i \rho)\right| \asymp \delta,
$$

and let $w_{t, x}(s)$ denote the characteristic

$$
w=w(s ; t, x+i \rho(t, x)) .
$$

By (1.19), (1.20), and (1.23), we obtain the following system of equations:

$$
\begin{aligned}
\log \frac{\Im\left[w_{t, x}(0)\right]}{\rho(t, x)} & =\int_{0}^{t} M\left(s, w_{t, x}(s)\right) d s, \\
\log \frac{\delta \Im\left[w_{t, x}(0)\right]}{\rho^{2}(t, x)} & =\int_{0}^{t} K\left(s, w_{t, x}(s)\right) d s+O(1) .
\end{aligned}
$$

The main observation is that $M\left(s, w_{t, x}(s)\right) \asymp K\left(s, w_{t, x}(s)\right)$, unless the measure $\mu_{s}$ is "concentrated" near the point $\Re\left[w_{t, x}(s)\right]$. The positivity of $K$ implies a Beurlingtype bound for $\rho$, but we get a better estimate for non-concentrated growth measures. This argument can be used to prove results similar to the estimate (1.16), which essentially states that growth measures are not concentrated with respect to most of the phase flow trajectories. Moreover, the method applies to arbitrary evolution processes related to first order PDEs as long as the corresponding kernels have suitable localization properties. 
1.5. Non-analytic chains. In the second part of the paper, we illustrate this real-variable approach by applying it to a system which is similar to $(1.24)-(1.25)$ but has a lower dimensional phase space. Roughly speaking, we consider only the imaginary parts of characteristics assuming that the real parts remain constant. We establish an analogue of (1.16), see Theorem 3.1, and derive the following growth estimate for the corresponding version of an $\operatorname{HS}(\delta)$-process.

Let $\mu_{t}$ be a family of positive measures on $\mathbb{R}$ periodic with period 1 . Define the kernels $M$ and $K$ by the formulae (1.21) and (1.22) respectively. Given a positive function $\rho=\rho(t, x)$, for each $(t, x)$ let $y(s)=y_{t, x}(s)$ denote the solution of the initial value problem

$$
\partial_{s} y=-y M(s, x+i y), \quad y(t)=\rho(t, x)
$$

Define the times $T_{0}$ and $T$ by the equations

$$
\int_{0}^{T_{0}} \mu_{t}[0,1] d t=\int_{T_{0}}^{T} \mu_{t}[0,1] d t=\frac{1}{2}
$$

Theorem B. Suppose that for all $t \geq T_{0}$, we have

$$
\begin{gathered}
\log \frac{\delta y_{t, x}(0)}{\rho^{2}(t, x)}=\int_{0}^{t} K\left(s, x+i y_{t, x}(s)\right) d s+O(1), \\
d \mu_{t}(x)=\rho_{t}^{2}(t, x) d x .
\end{gathered}
$$

Then $T-T_{0} \gtrsim \delta^{-(1+c)}$ for some absolute constant $c>0$.

The system (1.26) - (1.27) is of course an analogue of (1.24) - (1.25). Together with (1.28) it describes an evolution process corresponding to the family of nonanalytic maps

$$
\psi_{t}: x+i y \mapsto x+i \beta(t, x, y), \quad \psi_{0}=\mathrm{id},
$$

with $\beta=\beta(t, x, y)$ satisfying the partial differential equation

$$
\partial_{t} \beta=P \partial_{y} \beta,
$$

where $P=P(t, x, y)$ is the Poisson integral of the function $\rho(t, \cdot)^{2}$ such that

$$
\rho\left|\partial_{y} \beta(t, x, \rho)\right| \asymp \delta, \quad \rho=\rho(t, x) .
$$

The latter is similar to the relation (1.23). The PDE (1.29) should be compared to the equation

$$
\partial_{t} \beta=P \partial_{y} \beta+\tilde{P} \partial_{x} \beta
$$

( $\tilde{P}$ is the conjugate Poisson integral), which is equivalent to the analytic Loewner equation (take the imaginary part in (1.3) and denote $\beta:=\Im \varphi_{t}$ ). The complex analytic method of Section 2 does not apply to the system of Theorem B, and we don't know if Kesten's 3/2-result is valid in this case. 


\section{Deterministic Version of Kesten's theOREM}

In this section we prove the bound $T \lesssim \delta^{-3 / 2}$ for $\mathrm{HS}(\delta)$-chains (Theorem A). We also establish a stronger form of the Kesten-type estimate for Loewner chains with growth measures satisfying the equation

$$
d \mu_{t}(\zeta)=\left|\varphi_{t}^{\prime}(\zeta+\varepsilon \zeta)\right|^{-2}|d \zeta|, \quad(\varepsilon>0 \quad \text { fixed })
$$

2.1. Proof of Theorem A. Let $\varphi_{t}$ be an $\mathrm{HS}(\delta)$-process. Denote

$$
R(t):=\max \left\{\left|\varphi_{t}(\zeta)\right|: \zeta \in \partial \Delta\right\} .
$$

Assuming

$$
R(0) \asymp 1, \quad R(T)-R(0) \asymp 1,
$$

we want to show that $T \lesssim \delta^{-3 / 2}$. Let $0 \leq t_{2}<t_{1} \leq T$, and suppose that

$$
R\left(t_{1}\right)-R\left(t_{2}\right)>10 \delta .
$$

Let us choose a point $a$ such that

$$
|a|=R\left(t_{1}\right)+\delta, \quad \operatorname{dist}\left(a, \partial \Omega_{t_{1}}\right)=\delta,
$$

and define $t_{0}$ to be the first time when the cluster intersects the disc of radius $10 \delta$ centered at $a$. Clearly, $t_{0}>t_{2}$. To prove the theorem, it will be sufficient to show that

$$
t_{1}-t_{0} \gtrsim \delta^{-1 / 2}
$$

We will use the inverse Loewner equation

$$
\frac{\dot{z}(s)}{z(s)}=-\int \frac{z(s)+\zeta}{z(s)-\zeta} d \mu_{s}(\zeta)
$$

for the function

$$
z(s):=\varphi_{s}^{-1}(a), \quad\left(t_{0} \leq s \leq t_{1}\right)
$$

If we denote

$$
y(s):=|z(s)|-1,
$$

then the equation implies that

$$
-\frac{\dot{y}(s)}{y(s)} \asymp \int \frac{d \mu_{s}(\zeta)}{|z(s)-\zeta|^{2}} .
$$

Lemma. For all $s \in\left(t_{0}, t_{1}\right)$, we have

$$
\int \frac{d \mu_{s}(\zeta)}{|z(s)-\zeta|^{2}} \lesssim \sqrt{\delta}
$$

Assuming this fact, we can finish the proof as follows. Observe that

$$
\log \frac{y\left(t_{0}\right)}{y\left(t_{1}\right)} \gtrsim 1
$$

Indeed, let $G_{1}$ and $G_{0}$ be the Green functions of the domains $\Omega_{t_{1}}$ and $\Omega_{t_{0}}$ respectively, with pole at infinity. By the comparison principle, we have

$$
G_{0} \geq G_{1}
$$


Consider the disc $B:=B(a, 5 \delta)$, and let $U$ be the component of $\Omega_{t_{1}} \cap B$ containing a. Define

$$
\alpha:=\partial U \cap \partial B, \quad \beta:=\partial U \cap B .
$$

If $\omega$ denotes the harmonic measure of $U$ evaluated at $a$, then we have

$$
\begin{gathered}
G_{1}(a)=\int_{\alpha} G_{1} d \omega \\
G_{0}(a)=\int_{\alpha} G_{0} d \omega+\int_{\beta} G_{0} d \omega .
\end{gathered}
$$

By Beurling's projection theorem, we have

$$
\omega(\beta)>1 / 2,
$$

and by Harnack's inequality,

$$
G_{0}(\cdot) \asymp G_{0}(a) \text { on } \beta \text {. }
$$

It follows that

$$
\int_{\beta} G_{0} d \omega \geq c G_{0}(a)
$$

with an absolute constant $c \in(0,1)$, and therefore

$$
(1-c) G_{0}(a) \geq \int_{\alpha} G_{0} d \omega \geq \int_{\alpha} G_{1} d \omega=G_{1}(a) .
$$

Thus we have

$$
\log \frac{G_{0}(a)}{G_{1}(a)} \gtrsim 1
$$

which is equivalent to (2.4). The estimate (2.1) now follows from $(2.2)-(2.4)$ :

$$
\begin{aligned}
1 \lesssim \log \frac{y\left(t_{0}\right)}{y\left(t_{1}\right)} & =\int_{t_{1}}^{t_{0}} \frac{\dot{y}}{y} \\
& =\int_{t_{0}}^{t_{1}} d s \int \frac{d \mu_{s}(\zeta)}{|z(s)-\zeta|^{2}} \lesssim\left(t_{1}-t_{0}\right) \sqrt{\delta} .
\end{aligned}
$$

This completes the proof of Theorem A except that we still need to prove the lemma.

2.2. Proof of Lemma. The argument is based on the estimate (1.16), which we apply in the following form:

Let $\psi \in(\Sigma)$ and $0<\sigma<1$. Suppose that a function $\rho(\eta)$ on the unit circle is such that

$$
\rho\left|\psi^{\prime}(\eta+\rho \eta)\right| \leq \sigma \quad(\rho:=\rho(\eta), \eta \in \partial \Delta) .
$$

Then for some absolute constant $c>0$, we have

$$
\int \rho^{2}(\eta)|d \eta| \lesssim \sigma^{1+c} .
$$

Let us fix $s \in\left(t_{0}, t_{1}\right)$ and drop the index $s$ from the notation $\Omega_{s}, \varphi_{s}$, $\varepsilon_{s}$; in particular, we have

$$
d \mu_{s}(\zeta)=\varepsilon^{2}(\zeta)|d \zeta|
$$


We will also write $y$ for $y(s)$ and we will assume that $z(s)=1+y$. Moving the point $a$ within a disc of a fixed hyperbolic radius and applying Harnack's inequality, we can assume without loss of generality that

$$
y \asymp \varepsilon(1) .
$$

For $0 \leq n \lesssim|\log \varepsilon|$, we denote

$$
y_{n}=2^{n} y
$$

and consider the intervals $I_{n} \subset \partial \Delta$ of length $y_{n}$ centered at 1 . Then we have

$$
\int_{\partial \Delta} \frac{d \mu_{s}(\zeta)}{|z(s)-\zeta|^{2}}=\int_{I_{0}}+\sum_{n \geq 0} \int_{I_{n+1} \backslash I_{n}} \lesssim \sum_{n \geq 0} \frac{1}{y_{n}^{2}} \int_{I_{n}} \varepsilon^{2}(\zeta)|d \zeta| .
$$

We also denote

and observe that

$$
r_{n}=1+y_{n}, \quad d_{n}=y_{n}\left|\varphi^{\prime}\left(r_{n}\right)\right|
$$

$$
d_{n} \asymp \operatorname{dist}\left(\varphi\left(r_{n}\right), \partial \Omega\right), \quad d_{0} \asymp \delta
$$

We claim that

$$
\left|\varphi^{\prime}(1+y)\right| \lesssim\left|\varphi^{\prime}\left(r_{n}\right)\right|
$$

and

$$
\int_{I_{n}} \varepsilon^{2}(\zeta)|d \zeta| \lesssim y_{n}^{3}\left(\frac{\delta}{d_{n}}\right)^{1+c}
$$

where the constant $c$ is the same as in (2.5). The statement of the lemma is a consequence of these two facts. Indeed, by (2.6) and (2.8) we have

$$
\int \frac{d \mu_{s}(\zeta)}{|z(s)-\zeta|^{2}} \lesssim \sum y_{n}\left(\frac{\delta}{d_{n}}\right)^{1+c} \lesssim y \sum\left(\frac{\delta}{d_{n}}\right)^{c}
$$

where the last inequality follows from the distortion theorem and (2.7):

$$
\frac{y_{n}}{d_{n}} \lesssim \frac{y}{\delta}
$$

For the same reason, the last sum in (2.9) is finite, and therefore

$$
\int \frac{d \mu_{s}(\zeta)}{|z(s)-\zeta|^{2}} \lesssim y \lesssim \sqrt{\delta}
$$

To verify $(2.7)$ and $(2.8)$, we denote by $w_{n}$ the point in $\Delta$ such that

$$
\varphi\left(w_{n}\right)=\left(1+d_{n}\right) a .
$$

By construction, the halfline $\mathbb{R}_{+} a$ lies in a conformal Stoltz angle of the domain $\Omega$, and therefore the hyperbolic distance between the points $r_{n}$ and $w_{n}$ is bounded from above by a universal constant. Let us fix $n$, and consider the conformal automorphism $\tau: \Delta \rightarrow \Delta$ given by the formula

$$
\tau(w)=\frac{w_{n} w+1}{w+w_{n}},
$$

so that $\infty \mapsto w_{n}$ and $1 \mapsto 1$. Let $\psi \in(\Sigma)$ be the corresponding Möbius transform of the function $\varphi$ :

$$
\psi:=\frac{\left(1-w_{n}^{2}\right) \varphi^{\prime}\left(w_{n}\right)}{\varphi \circ \tau-\varphi\left(w_{n}\right)}=T \circ \varphi \circ \tau
$$


where

$$
T(z)=\frac{\left(1-w_{n}^{2}\right) \varphi^{\prime}\left(w_{n}\right)}{z-\varphi\left(w_{n}\right)}
$$

is the conformal map from $\Omega$ onto the domain $\tilde{\Omega}:=\psi \Delta$.

Let us prove (2.7). Denote by $w$ the preimage of the point $1+y$ under $\tau$. Then we have

$$
\begin{aligned}
\left|\psi^{\prime}(w)\right| & =\left|T^{\prime}(a) \varphi^{\prime}(1+y) \tau^{\prime}(w)\right| \\
& \asymp \frac{d_{n}}{\left|\varphi\left(w_{n}\right)-a\right|^{2}}\left|\varphi^{\prime}(1+y)\right| y_{n} \\
& \asymp d_{n}^{-1}\left|\varphi^{\prime}(1+y)\right| y_{n}=\frac{\left|\varphi^{\prime}(1+y)\right|}{\left|\varphi^{\prime}\left(r_{n}\right)\right|},
\end{aligned}
$$

and so we need to show that $\left|\psi^{\prime}(w)\right| \lesssim 1$. To see this, denote

$$
U:=\left\{|z|>R\left(t_{1}\right)\right\}, \quad \tilde{U}:=T U .
$$

Clearly,

$$
\operatorname{dist}(a, \partial U) \asymp \operatorname{dist}(a, \partial \Omega),
$$

and applying the map $T$, we have

$$
\operatorname{dist}(T a, \partial \tilde{U}) \asymp \operatorname{dist}(a, \partial \tilde{\Omega}) .
$$

By the comparison principle, we have the inequality

$$
G_{\tilde{U}}(\cdot) \leq G_{\tilde{\Omega}}(\cdot)
$$

for Green's functions with pole at infinity. It follows that

$$
\begin{aligned}
\left|\psi^{\prime}(w)\right| & \asymp \frac{\operatorname{dist}(\psi(w), \partial \tilde{\Omega})}{|w|-1} \asymp \frac{\operatorname{dist}(T a, \partial \tilde{\Omega})}{G_{\tilde{\Omega}}(T a)} \\
& \lesssim \frac{\operatorname{dist}(T a, \partial \tilde{U})}{G_{\tilde{U}}(T a)} \asymp 1 ;
\end{aligned}
$$

the latter holds since $\partial \tilde{U}$ is a round circle of radius $\asymp 1$.

Finally, let us prove (2.8). Define the function $\rho(\eta)$ by the equation

$$
\rho(\eta)=y_{n}^{-1} \varepsilon(\tau \eta), \quad(\eta \in \partial \Delta)
$$

We will see that

$$
\operatorname{dist}(\psi(\eta+\rho(\eta) \eta), \partial \tilde{\Omega}) \lesssim \frac{\delta}{d_{n}} .
$$

It then follows from the estimate (2.5) with $\sigma:=d_{n}^{-1} \delta$ that

$$
\int \rho^{2}(\eta)|d \eta| \lesssim\left(\frac{\delta}{d_{n}}\right)^{1+c}
$$

Since

$$
\left|\left(\tau^{-1}\right)^{\prime}\right| \asymp y_{n}^{-1} \quad \text { on } \quad I_{n}
$$

the left hand side of the last inequality does not exceed

$$
\int_{\tau^{-1} I_{n}} \rho^{2}(\eta)|d \eta|=\frac{1}{y_{n}^{2}} \int_{I_{n}} \varepsilon^{2}(\zeta)\left|\left(\tau^{-1}\right)^{\prime}(\zeta)\right||d \zeta| \asymp \frac{1}{y_{n}^{3}} \int_{I_{n}} \varepsilon^{2}(\zeta)|d \zeta|
$$


which implies (2.8).

It remains to prove (2.10). For $\eta \in \tau^{-1} I_{n}$, denote

$$
\zeta:=\tau \eta, \quad z:=\tau(\eta+\rho(\eta) \eta) .
$$

Since $T \varphi(z)=\psi(\eta+\rho(\eta) \eta)$, by the distortion theorem we have

$$
\operatorname{dist}(\psi(\eta+\rho(\eta) \eta), \partial \tilde{\Omega}) \asymp\left|T^{\prime}(\varphi(z))\right| \operatorname{dist}(\varphi(z), \partial \Omega) .
$$

The hyperbolic distance between the points $z$ and $\zeta+\varepsilon(\zeta) \zeta$ is bounded by an absolute constant, which implies that

$$
\operatorname{dist}(\varphi(z), \partial \Omega) \asymp \operatorname{dist}(\varphi(\zeta+\varepsilon(\zeta) \zeta), \partial \Omega) \asymp \delta
$$

by the definition of $\varepsilon(\zeta)$. On the other hand, we have

$$
\left|\varphi\left(w_{n}\right)-\varphi(z)\right| \geq \operatorname{dist}\left(\varphi\left(w_{n}\right), \partial \Omega\right)-O(\delta) \asymp d_{n},
$$

and

$$
\left|T^{\prime}(\varphi(z))\right| \asymp \frac{d_{n}}{\left|\varphi\left(w_{n}\right)-\varphi(z)\right|^{2}} \lesssim \frac{1}{d_{n}} .
$$

The estimate (2.8) now follows from (2.11) - (2.13).

2.3. A version of Theorem A. In the definition of an $\operatorname{HS}(\delta)$-process, the parameter $\delta$ was fixed, but the quantity $\varepsilon$ depended on $\zeta \in \partial \Delta$ and time $t$. We obtain a somewhat simpler process if we keep $\varepsilon>0$ fixed and define the growth measures by the equation

$$
d \mu_{t}(\zeta)=\left|\varphi_{t}^{\prime}(\zeta+\varepsilon \zeta)\right|^{-2}|d \zeta|
$$

For such processes, we can establish a slightly stronger form of the Kesten-type estimate by working with the direct Loewner equation (1.3).

Theorem. Let $\varepsilon<1$ be a positive number, and suppose that the growth measures $\mu_{t}$ of a Loewner chain $\left\{\varphi_{t}\right\}$ satisfy (2.14). Denote

$$
R(t):=\max \left\{\left|\varphi_{t}(r \zeta)\right|: \zeta \in \partial \Delta\right\} \quad(r:=1+\varepsilon) .
$$

Then there is an absolute constant $C$ such that if $R(t) \leq 1$, then

$$
\limsup _{\Delta t \rightarrow 0} \frac{R(t+\Delta t)-R(t)}{\Delta t} \leq C \varepsilon^{-1} .
$$

It is perhaps interesting to mention that a similar estimate $\dot{c}(t) \lesssim \varepsilon^{-1}$ for the logarithmic capacity is not clear at all. By (1.4), the latter is essentially equivalent to the Brennan conjecture [4]:

$$
\int_{\partial \Delta}\left|\psi^{\prime}(r \zeta)\right|^{-2}|d \zeta| \lesssim \frac{1}{r-1}, \quad \forall \psi \in(\Sigma) .
$$

The inequality (2.15) is of course best possible, as the case of the function $\varphi_{0}(z)=$ $z+z^{-1}$ shows. The theorem implies the bound $T \gtrsim \varepsilon$ for the doubling time defined by equations (1.13).

Proof: For each $\zeta \in \partial \Delta$, the function $t \mapsto R_{\zeta}(t):=\left|\varphi_{t}(r \zeta)\right|$ is differentiable, and there is a uniform upper bound for the derivative, e.g. $\dot{R}_{\zeta}(t) \lesssim \varepsilon^{-3}$. It follows that to prove (2.15) it is enough to show that for any fixed $t$, we have

$$
\dot{R}_{\zeta_{*}}(t) \lesssim \varepsilon^{-1}
$$


where $\zeta_{*}$ is a point at which the function $\zeta \mapsto R_{\zeta}(t)$ attains its maximum value. To this end, let us rewrite the Loewner equation in the form

$$
\frac{\dot{\varphi}_{t}(z)}{\varphi_{t}(z)}=\frac{z \varphi_{t}^{\prime}(z)}{\varphi_{t}(z)} \int \frac{z+\zeta}{z-\zeta} d \mu_{t}(\zeta)
$$

and note that

$$
\frac{z \varphi_{t}^{\prime}(z)}{\varphi_{t}(z)} \in \mathbb{R} \quad \text { at } \quad z=r \zeta_{*} .
$$

Taking the real part in (2.16), we have

$$
\dot{R}_{\zeta_{*}}(t) \lesssim\left|\varphi_{t}^{\prime}\left(r \zeta_{*}\right)\right| \int_{\partial \Delta} \frac{r^{2}-1}{\left|r \zeta_{*}-\zeta\right|^{2}}\left|\varphi_{t}^{\prime}(r \zeta)\right|^{-2}|d \zeta| \lesssim \frac{1}{\varepsilon}
$$

The second inequality in (2.17) is an analogue of (2.3) and can be established by the same method. Namely, let us assume that $\zeta_{*}=1$ and write $\varphi$ for $\varphi_{t}$. If we consider the intervals $I_{n} \subset \partial \Delta$ of length $\varepsilon 2^{n}$ centered at 1 with $n \geq 1$ and $2^{n} \lesssim 1 / \varepsilon$, then the middle expression in (2.17) does not exceed

$$
\left|\varphi^{\prime}(r)\right| \sum_{(n)} \frac{\varepsilon}{\left|I_{n}\right|^{2}} \int_{I_{n}}\left|\varphi^{\prime}(r \zeta)\right|^{-2}|d \zeta| .
$$

Denote $r_{n}=1+\varepsilon 2^{n}$ and observe that for all $\mathrm{n}$, we have

$$
\left|\varphi^{\prime}(r)\right| \lesssim\left|\varphi^{\prime}\left(r_{n}\right)\right|
$$

and

$$
\frac{1}{\left|I_{n}\right|} \int_{I_{n}}\left|\varphi^{\prime}(r \zeta)\right|^{-2}|d \zeta| \lesssim \frac{1}{\left|\varphi^{\prime}\left(r_{n}\right)\right|^{2}}\left(\frac{r_{n}-1}{r-1}\right)^{q}
$$

with an absolute constant $q<2$. It follows that

$$
\begin{aligned}
(2.18) & \lesssim\left|\varphi^{\prime}(r)\right| \sum_{(n)} \frac{\varepsilon}{\left|I_{n}\right|} \frac{1}{\left|\varphi^{\prime}\left(r_{n}\right)\right|^{2}}\left(\frac{r_{n}-1}{r-1}\right)^{q} \\
& \lesssim \frac{1}{\varepsilon} \sum_{(n)} \frac{r_{n}-1}{\left|\varphi^{\prime}\left(r_{n}\right)\right|}\left(\frac{r_{n}-1}{r-1}\right)^{q-2} \lesssim \frac{1}{\varepsilon}
\end{aligned}
$$

The inequalities (2.19) and (2.20) can be verified as in the proof of (2.3) by considering the Möbius transform

$$
\psi=\frac{\left(1-r_{n}^{2}\right) \varphi^{\prime}\left(r_{n}\right)}{\varphi \circ \tau-\varphi\left(r_{n}\right)} \in(\Sigma)
$$

of the function $\varphi$, where $\tau$ is the conformal automorphism of $\Delta$ such that $\infty \mapsto$ $r_{n}, 1 \mapsto 1$. The constant $q$ in (2.20) comes from a universal estimate of integral means of the derivative in the class $(\Sigma)$ :

$$
\int_{\partial \Delta}\left|\psi^{\prime}(\rho \zeta)\right|^{-2}|d \zeta| \lesssim\left(\frac{1}{\rho-1}\right)^{1.9}, \quad(0<\rho<1),
$$

see [18], Chapter 8. 


\section{Estimates For the INVERSe Loewner EQUATion}

In this section, we discuss a real variable approach to growth estimates which is based on the study of the inverse Loewner chains, see Section 1.4. We will be considering the simplified, non-analytic model described in Section 1.5. Let us recall the equations for this model. We will use a slightly different notation.

Let $\left\{\mu_{t}\right\}, 0 \leq t \leq T$, be a family of finite positive measures on $\mathbb{T}:=\mathbb{R} / \mathbb{Z}$. Denote

$$
\Omega:=[0, T] \times \mathbb{T} .
$$

(The same symbol $\mu_{t}$ will be used for the periodic extension of $\mu_{t}$ to $\mathbb{R}$.) We have two measures on $\Omega$ - the area measure $A$, and the measure $\mu$ corresponding to the family $\left\{\mu_{t}\right\}$.

For each $x \in \mathbb{T}$, we define the functions

$$
\begin{aligned}
M_{x}(s, y) & :=\int_{\mathbb{R}} \frac{d \mu_{s}(\xi)}{(x-\xi)^{2}+y^{2}}, \\
K_{x}(s, y) & :=\int_{\mathbb{R}} \frac{(x-\xi)^{2} d \mu_{s}(\xi)}{\left[(x-\xi)^{2}+y^{2}\right]^{2}},
\end{aligned}
$$

where $0 \leq s \leq T$ and $y>0$, and we consider the following family ordinary differential equations:

$$
\frac{\dot{y}}{y}=-M_{x}(s, y)
$$

Every positive solution $y=y(s)$ of $\left(E_{x}\right)$ is monotone decreasing. We specify the initial conditions as follows. Let $\rho=\rho(\omega)$ be a given a positive function on $\Omega$. Then for each $\omega=(t, x)$, we denote by $y_{\omega}(\cdot)$ the solution of $\left(E_{x}\right)$ satisfying

$$
y_{\omega}(t)=\rho(\omega) .
$$

This solution exists on the interval $[0, t]$, and we define

$$
Y(\omega):=y_{\omega}(0)
$$

Our goal is to prove the following statement.

3.1. Theorem. Let $\mu$ be a probability measure and let $\rho$ be a positive function on $\Omega=[0, T] \times \mathbb{T}$. Suppose that for some $\delta \in(0,1)$, the following inequality holds for all points $\omega=(t, x) \in \Omega$ :

$$
\log \frac{\delta Y(\omega)}{\rho^{2}(\omega)} \geq \int_{0}^{t} K_{x}\left(s, y_{\omega}(s)\right) d s
$$

Then, for some absolute constant $c>0$, either

$$
\mu\left\{\rho \leq \delta^{\frac{1+c}{2}}\right\}>\frac{1}{2}
$$

or there is a subset $\Omega^{\prime} \subset \Omega$ satisfying

$$
\mu\left(\Omega^{\prime}\right)>c, \quad A\left(\Omega^{\prime}\right)<\delta^{c} A(\Omega) .
$$


Observe that for all $\omega \in \Omega$, we have

$$
Y(\omega) \leq 2
$$

To see this, note that $M_{x}(s, y) \leq y^{-2}\left\|\mu_{s}\right\|$, and therefore if $\omega=(t, x)$, then

$$
\partial_{s} y_{\omega}^{2}(s) \geq-\left\|\mu_{s}\right\|, \quad(0 \leq s \leq t) .
$$

On the other hand, since $K_{x} \geq 0$, it follows from (3.1) that

$$
\rho^{2}(\omega) \leq \delta Y(\omega)
$$

Since $\mu$ is a probability measure, we have

$$
Y^{2}(\omega) \leq 2+\rho^{2}(\omega) \leq 2+\delta Y(\omega)
$$

which implies (3.4) for $\delta<1$. From (3.4) and (3.5), we obtain a Beurling-type estimate: $\rho(\omega) \lesssim \sqrt{\delta}$. Our theorem states that $\rho$ can not be too close to the upper bound $\sqrt{\delta}$ on a set of large $\mu$-measure unless $\mu$ is "very singular" with respect to the area measure. The following two simple examples illustrate the dichotomy (3.2) $-(3.3)$.

(i) Let $\mu$ be the area measure on $\Omega=[0,1] \times \mathbb{T}$. Then we have

$$
K_{x} \geq \frac{1}{2} M_{x}, \quad(\forall x \in \mathbb{T}),
$$

and so (3.1) implies

$$
\frac{\delta Y}{\rho^{2}} \geq \sqrt{\frac{Y}{\rho}}, \quad(\forall \omega \in \Omega) .
$$

In particular, by (3.4) we must have $\rho \lesssim \delta^{2 / 3}$. We can actually get $\rho \asymp \delta^{2 / 3}$ on a set of large measure - it is easy to show that $(3.1)$ holds for $\rho(t, x)=$ const $(\delta t)^{2 / 3}$ provided that the constant is sufficiently small.

(ii) Let $\mu$ be the linear measure of the set $[0,1] \times\left\{x_{0}\right\}$ and let $\rho \equiv \delta^{1 / 2}$ on this set and be zero elsewhere. Then (3.1) is clearly satisfied and the bound (3.5) is attained on a set of full measure.

Theorem 3.1 implies the following estimate of the "doubling" time $T$.

Corollary. Suppose $\mu$ and $\rho$ satisfy the hypothesis of the theorem, and suppose that they are related by the equation $d \mu=\rho^{2} d A$. Then $T \gtrsim \delta^{-(1+c)}$.

Indeed, applying Theorem 3.1 we see that if (3.2) holds, then

$$
\frac{1}{2} \leq \int_{\Omega} \delta^{1+c} d A \leq T \delta^{1+c},
$$

and if (3.3) holds, then we have

$$
c \leq \int_{\Omega^{\prime}} \rho^{2} d A \leq \delta A\left(\Omega^{\prime}\right) \leq T \delta^{1+c} .
$$

Theorem B (see Introduction) is a version of the last statement. It will be clear from the proof of Theorem 3.1 that the changes in the argument are quite obvious.

We now turn to the proof of Theorem 3.1. Our construction depends on a certain numerical parameter $\varepsilon$ which will be specified at the end of the argument. By the 
notation of the type $a \lesssim b$ we mean the inequality $a \leq$ const $b$ with an absolute constant independent of the choice of $\varepsilon ; a \asymp b$ means that $a \lesssim b$ and $a \gtrsim b$.

3.2. Martingale structure. We first introduce a certain martingale structure in the set $\Omega$.

Lemma 1. There exists a sequence of partitions $\left\{\Pi_{j}\right\}_{j \geq 1}$ of $\Omega$ satisfying the following conditions.

- Each partition $\Pi_{j}$ consists of rectangles $P=I \times l$, where $l \subset \mathbb{T}$ is a dyadic interval of length $2^{-j}$ and $I$ is a subinterval of $[0, T]$.

- Rectangles of the same rank $j$ have disjoint interiors. Each $P \in \Pi_{j}$ is a union of rectangles of rank $j+1$.

- If $P=I \times l \in \Pi_{j}$ and if $x_{l}$ is the center of $l$, then

$$
\frac{1}{4} \leq \int_{I} d s \int_{\mathbb{T}} \frac{d \mu_{s}(\xi)}{\left(x_{l}-\xi\right)^{2}+4^{-j}} \leq 4 .
$$

Proof: Denote

$$
M_{x}^{j}(s):=\int_{\mathbb{T}} \frac{d \mu_{s}(\xi)}{\left(x_{l}-\xi\right)^{2}+4^{-j}} .
$$

Clearly, $M_{x}^{j} \leq M_{x}^{j+1}$, and it is easy to see that

$$
\left|x^{\prime}-x\right| \leq 2^{-j-1} \quad \Rightarrow \quad \frac{1}{2} M_{x}^{j} \leq M_{x^{\prime}}^{j} \leq 2 M_{x}^{j} .
$$

We begin by setting $P_{0}=\Omega$. Suppose a rectangle $P_{j}=I \times l$ of rank $j$ is already constructed and suppose that

$$
\frac{1}{2}<\int_{I} M_{x_{l}}^{j} \leq 1 .
$$

Note that (3.8) holds for $P_{0}$. Let $k>j$ be the least integer such that

$$
\int_{I} M_{x_{l}}^{k}>2
$$

We first subdivide $P_{j}$ successively into rectangles of ranks $j+1, \ldots, k-1$ with the same $t$-projection $I$ as $P_{j}$. By (3.8) and (3.7), the new rectangles satisfy (3.6). Then we subdivide each of the rectangles of rank $k-1$ already obtained into rectangles of rank $k$ satisfying (3.8). The latter is possible by (3.7).

We fix partitions $\Pi_{j}$ constructed in Lemma 1 for the rest of the proof. If $\omega \in \Omega$, then we write $P_{j}(\omega)$ for the element of $\Pi_{j}$ containing $\omega$. It is clear from (3.6) that

$$
P \in \Pi_{j} \Rightarrow \mu(P) \lesssim 4^{-j}
$$

Another property of the construction is stated in the following lemma, the proof of which we leave as a simple exercise.

Lemma 2. Let $P=I \times l \in \Pi_{j}, x \in l$, and let $y(\cdot)$ be a solution of $\left(E_{x}\right)$. Then

(i) if $y\left(s_{1}\right) \geq 2^{-j+10}$ for some $s_{1} \in I$, then $y(s) \geq 2^{-j}$ for all $s \in I$;

(ii) if $y\left(s_{1}\right) \asymp 2^{-j}$ for $s_{1} \in I$, then $y(s) \lesssim 2^{-j}$ for all $s \in I$, and $y\left(t_{-}\right) / y\left(t_{+}\right)-$ $1 \gtrsim 1$, where $t_{-}<t_{+}$are the endpoints of $I$. 
3.3. Rectangles $R_{j}(\omega)$. Let $\omega=(t, x) \in \Omega$ and let $y_{\omega}(\cdot)$ be the corresponding solution of $\left(E_{x}\right)$. We now construct certain rectangles associated with this solution. Suppose that a positive integer $j$ satisfies

$$
\rho(\omega) \leq 2^{-j-10} \leq Y(\omega) .
$$

Then we define $R_{j}(\omega)$ as a rectangle $I \times l \in \Pi_{j}$ such that

$$
y_{\omega}(s)=2^{-j+10} \quad \text { for some } \quad s \in I .
$$

We don't define rectangles $R_{j}(\omega)$ for other values of $j$. Let $I_{j}(\omega)$ denote the projection of $R_{j}(\omega)$ to the $t$-axis. It follows from Lemma 2 that

$$
y_{\omega}(s) \asymp 2^{-j}, \quad\left(\forall s \in I_{j}(\omega)\right),
$$

and that the intervals $I_{j}(\omega)$ with the same $\omega$ are essentially disjoint:

$$
|k-j|>10 \quad \Rightarrow \quad I_{k}(\omega) \cap I_{j}(\omega)=\emptyset .
$$

We will also need the following fact.

Lemma 3. If $P \in \Pi_{j}$, then $\mu\left\{\omega: R_{j}(\omega)=P\right\} \lesssim 4^{-j}$.

Proof: Let $P=\left(s_{-}, s_{+}\right) \times l \in \Pi_{j}$, and $S=\left\{\omega: R_{j}(\omega)=P\right\}$. For each point $\omega=(t, x) \in S$ with $x \in l$, we denote by $\mathcal{C}_{\omega}$ the family of rectangles $Q$ satisfying the following condition:

$$
\exists s, \quad s_{+} \leq s \leq t, \quad y_{\omega}(s) \asymp 2^{-k}, \quad k:=\operatorname{rank} Q>j, \quad(s, x) \in Q .
$$

Define

$$
\mathcal{C}_{*}:=\bigcup\left\{\mathcal{C}_{\omega}: \omega \in S\right\}
$$

and consider a subcollection $\mathcal{C} \subset \mathcal{C}_{*}$ constructed as follows. We first take rectangles $Q \in \mathcal{C}_{*}$ of rank $j+1$, then we add rectangles of rank $j+2$ which are not contained in any $\mathcal{C}_{*}$-rectangle of rank $j+1$, then we add rectangles of rank $j+3$ which are not contained in any rectangle of rank $j+1$ or $j+2$ etc. Clearly,

$$
S \subset \bigcup\{Q: Q \in \mathcal{C}\},
$$

and so it is sufficient to show that

$$
\sum_{Q \in \mathcal{C}} \mu Q \lesssim 4^{-j}
$$

One of the properties of the family $\mathcal{C}$ is that the number of rectangles $Q_{k} \in \mathcal{C}$ of rank $k$ intersecting any given dyadic strip $\left\{x_{1} \leq x \leq x_{2}\right\}$ of width $2^{-k}$ is bounded by an absolute constant. To see this, consider the rank $k$ rectangle with the largest $t$-coordinate $t_{*}$ of the center, and let $\omega \in S$ be such that $y_{\omega}\left(t_{*}\right) \asymp 2^{-k}$. By construction, the solution $y_{\omega}$ must be $\asymp 2^{-k}$ in all other rectangles $Q_{k}$, because otherwise $Q_{k}$ would be covered by a $\mathcal{C}$-rectangle of a smaller rank. It remains to note that by Lemma 2, the function $y_{\omega}$ drops by a constant factor across every rectangle $Q_{k}$. It follows that

$$
N_{k}:=\#\{Q \in \mathcal{C}: \operatorname{rank} Q=k\} \lesssim 2^{k-j}
$$

and therefore by (3.9), we have

$$
\sum_{Q \in \mathcal{C}} \mu Q \lesssim \sum_{k \geq j} N_{k} 4^{-k} \lesssim 4^{-j}
$$


3.4. Concentrated rectangles. We will write $K_{\omega}(s)$ for the function $s \mapsto K_{x}\left(s, y_{\omega}(s)\right)$. The notation $M_{\omega}(s)$ has the similar meaning. Our next goal is to estimate the integral of $K_{\omega}$ over $I_{j}(\omega)$. Note that by $(3.6)$ and $(3.10)$, we have

$$
\int_{I_{j}(\omega)} M_{\omega}(s) d s \geq 2 a
$$

for some absolute constant $a>0$.

Fix a small dyadic number $\varepsilon>0$ to be specified later. The following definitions depend on the choice of $\varepsilon$. A rectangle $P=I \times l \in \Pi_{j}$ is said to be concentrated if there is a subinterval $l^{\prime} \subset l$ of length

$$
\left|l^{\prime}\right|=\frac{\varepsilon}{10}|l|
$$

such that for $P^{\prime}:=I \times l^{\prime}$ we have

$$
\mu P^{\prime}>a 4^{-j}, \quad \mu\left(P \backslash P^{\prime}\right)<\frac{a}{10} 4^{-j} .
$$

In this case, we can choose some interval of concentration $\lambda(P) \subset l$ of length $\varepsilon 2^{-j}$ so that it consists of two adjacent dyadic intervals and covers

the $\left|l^{\prime}\right|$-neighborhood of $l^{\prime}$. Note that if $l^{\prime \prime}$ is an interval with the same properties as $l^{\prime}$, then $\lambda(P)$ covers $l^{\prime \prime}$ as well. For non-concentrated rectangles $P$, we simply set $\lambda(P)=\emptyset$. Finally, we define

$$
\hat{P}=I \times \lambda(P) .
$$

Lemma 4. Suppose a rectangle $R_{j}(\omega)=I_{j}(\omega) \times l$ is defined for some $\omega=(t, x)$. Also suppose that

$$
\operatorname{dist}(x, \mathbb{T} \backslash l)>\varepsilon|l| \text {. }
$$

Then either $R_{j}(\omega)$ is concentrated and $x \in \lambda\left(R_{j}(\omega)\right)$, or

$$
\int_{I_{j}(\omega)} K_{\omega} \geq \alpha
$$

where $\alpha=\alpha(\varepsilon)$ is a positive number depending on the choice of $\varepsilon$.

Proof: Let $l^{\prime}$ denote the interval of length $10^{-1} \varepsilon|l|$ centered at $x$. Then $l^{\prime} \subset l$ by (3.14). We have

$$
\begin{aligned}
\int_{I_{j}(\omega)} K_{\omega} & \geq \int_{I_{j}(\omega)} d s \int_{l \backslash l^{\prime}} \frac{(x-\xi)^{2} d \mu_{s}(\xi)}{\left[(x-\xi)^{2}+y_{\omega}^{2}(s)\right]^{2}} \\
& \gtrsim \varepsilon^{2} \int_{I_{j}(\omega)} d s \int_{l \backslash l^{\prime}} \frac{d \mu_{s}(\xi)}{(x-\xi)^{2}+y_{\omega}^{2}(s)}
\end{aligned}
$$

It follows that if (3.15) does not hold with $\alpha \ll \varepsilon^{2}$, then the latter iterated integral is $\ll 1$, and therefore (3.12) implies the estimate

$$
\int_{I_{j}(\omega)} d s \int_{l^{\prime}} \frac{d \mu_{s}(\xi)}{(x-\xi)^{2}+y_{\omega}^{2}(s)} \geq a .
$$

Let us show that the inequalities (3.13) hold in this case, and so $R_{j}(\omega)$ is a concentrated rectangle. The first inequality follows from (3.16) and the fact that $y_{\omega}(\cdot)>2^{-j}$ on $I_{j}(\omega)$. To prove the second inequality in $(3.13)$, we observe that 


$$
\mu\left(R \backslash R^{\prime}\right) \asymp 4^{-j} \int_{I_{j}(\omega)} d s \int_{l \backslash l^{\prime}} \frac{d \mu_{s}(\xi)}{(x-\xi)^{2}+y_{\omega}^{2}(s)} \ll 4^{-j} .
$$

3.5. Proof of Theorem. Let $c<1 / 4$ be a positive number and

$$
E:=\left\{\omega: \rho(\omega)>\delta^{\frac{1+c}{2}}\right\} .
$$

If $\omega \in E$, then

$$
Y(\omega) \geq \frac{\rho^{2}(\omega)}{\delta}>\delta^{c},
$$

and since $\rho(\omega) \lesssim \sqrt{\delta}$, the rectangles $R_{j}(\omega)$ are defined for all $j \in[1+N, 2 N]$, where

For $\omega=(t, x) \in E$, denote

$$
N \asymp \log \frac{1}{\delta} .
$$

$$
n(\omega):=\#\left\{j \in[1+N, 2 N]: x \in \lambda\left(R_{j}(\omega)\right)\right\} .
$$

We also need the function

$$
N(\omega):=\#\left\{j \in[1+N, 2 N]: \omega \in \hat{P}_{j}(\omega)\right\}, \quad(\omega \in \Omega) .
$$

Define the sets

and

$$
E^{\prime}:=\left\{\omega \in E: n(\omega) \geq \frac{1}{4} N\right\}
$$

$$
\Omega^{\prime}:=\{\omega \in \Omega: N(\omega) \geq 2 \varepsilon N\} .
$$

Lemma 5. (i) There is a positive constant $c_{1}=c_{1}(\varepsilon)$ such that

$$
A\left(\Omega^{\prime}\right) \leq \delta^{c_{1}} A(\Omega) \text {. }
$$

(ii) There is an absolute constant $\varepsilon_{1}$ such that if $\varepsilon \leq \varepsilon_{1}$, then

$$
\mu E^{\prime} \geq \frac{1}{4} \Rightarrow \mu \Omega^{\prime} \gtrsim 1
$$

Proof: (i) Denote by $X_{j}$ the characteristic function of the set

$$
\bigcup\left\{\hat{P}: P \in \Pi_{j}\right\} .
$$

If $\varepsilon=2^{1-m}$, where $m$ is a positive integer, then we have the submartingale property

$$
E\left(X_{j+m}-\varepsilon \mid \Pi_{j}\right) \leq 0,
$$

where $E$ is the conditional expectation with respect to the normalized area measure. For $1 \leq \nu \leq m$, let

$$
\Omega_{\nu}^{\prime}:=\left\{\frac{m}{N} \sum_{j=0}^{\frac{N}{m}-1} X_{N+\nu+j m} \geq 2 \varepsilon\right\} .
$$

(We can assume that $m$ divides $N$.) Simple large deviation argument shows that

$$
\frac{A\left(\Omega_{\nu}^{\prime}\right)}{A(\Omega)} \leq q^{N / m}
$$

for some $q=q(\varepsilon)<1$. Since

$$
\Omega^{\prime} \subset \bigcup \Omega_{\nu}^{\prime},
$$


the statement follows.

(ii) Suppose $\mu E^{\prime} \geq \frac{1}{4}$. Denote $f(\omega):=N^{-1} N(\omega)$. Since $f \leq 1$, we have

$$
\mu \Omega^{\prime}=\mu\{f \geq 2 \varepsilon\} \geq \int f d \mu-\int_{\{f<2 \varepsilon\}} f d \mu \geq \int f d \mu-2 \varepsilon,
$$

and it is enough to show that

$$
\int f d \mu \gtrsim 1
$$

We prove (3.17) by the following computation:

$$
\begin{aligned}
\frac{1}{16} & \leq \frac{1}{4} \mu E^{\prime} \leq \frac{1}{N} \int_{E^{\prime}} n(\omega) d \mu(\omega) \\
& =\frac{1}{N} \sum_{j=N+1}^{2 N} \mu\left\{\omega=(t, x): x \in \lambda\left(R_{j}(\omega)\right)\right\} \\
& =\frac{1}{N} \sum_{j=N+1}^{2 N} \sum_{P \in \Pi_{j}} \mu\left\{\omega: R_{j}(\omega)=P, x \in \lambda(P)\right\} \\
& \lesssim \frac{1}{N} \sum_{j=N+1}^{2 N} \sum_{P \in \Pi_{j}} \mu \hat{P} \\
& =\int f d \mu .
\end{aligned}
$$

The inequality (3.18) follows from Lemma 3 , for if $P \in \Pi_{j}$ is a concentrated rectangle, then

$$
\mu P \asymp \mu \hat{P} \asymp 4^{-j}
$$

and therefore

$$
\mu\left\{\omega: R_{j}(\omega)=R, x \in \lambda(P)\right\} \lesssim \mu \hat{P}
$$

which is also (trivially) true for non-concentrated rectangles.

We can now finish the proof of the theorem. We need one more notation. For $x \in \mathbb{T}$, let $l_{j}(x)$ denote the dyadic interval of rank $j$ containing $x$, and let

$$
k(x):=\#\left\{j \in[1+N, 2 N]: \operatorname{dist}\left(x, \mathbb{T} \backslash l_{j}(x)\right)<\varepsilon 2^{-j}\right\} .
$$

Consider the set

$$
E^{\prime \prime}:=\left\{\omega=(t, x) \in E: k(x) \geq \frac{1}{4} N\right\} .
$$

As in the proof of Lemma 5, we show that

$$
A\left(E^{\prime \prime}\right) \leq \delta^{c_{2}} A(\Omega)
$$

for some positive $c_{2}=c_{2}(\varepsilon)$ provided that $\varepsilon \leq \varepsilon_{2}$ for some absolute constant $\varepsilon_{2}>0$. We can now take $\varepsilon=\min \left\{\varepsilon_{1}, \varepsilon_{2}\right\}$ and choose $c \ll \alpha(\varepsilon)$, see Lemma 4 , so that we have

$$
E \subset E^{\prime} \cup E^{\prime \prime}
$$


The latter can be verified as follows. If $\omega \in E$, then by (1.1) and (1.4), we have

$$
\int_{0}^{t} K_{\omega} \leq \log \frac{2 \delta}{\rho^{2}(\omega)} \lesssim c N
$$

On the other hand, since the intervals $I_{j}(\omega)$ are essentially disjoint, see (3.11), we have

$$
\begin{aligned}
\int_{0}^{t} K_{\omega} & \gtrsim \sum_{j=N+1}^{2 N} \int_{I_{j}(\omega)} K_{\omega} \\
& \geq[N-k(x)-n(\omega)] \alpha(\varepsilon), \quad(\text { by }(3.15)) .
\end{aligned}
$$

It follows that either $k(\omega)$ or $n(\omega)$ is $\geq N / 4$, and we get (3.20).

Suppose now that $\mu E \geq 1 / 2$. Then either $\mu\left(E^{\prime}\right) \geq 1 / 4$, in which case Lemma 5 implies the theorem; or $\mu\left(E^{\prime \prime}\right) \geq 1 / 4$, and then the theorem follows from (3.19).

\section{REFERENCES}

[1] Ahlfors, L.V.: Conformal invariants. McGraw-Hill, 1973

[2] Aleksandrov, I.A.: Parametric continuations in the theory of univalent functions. Moscow: "Nauka", 1976 (Russian)

[3] Barlow, M.T.: Fractals, and diffusion-limited aggregation. Bull. Sc. Math. 117, 161-169 (1993)

[4] Brennan, J.E.: The integrability of the derivative in conformal mapping. J. London Math. Soc. 18, 261-272 (1978)

[5] Carleson, L.: On the distortion of sets on a Jordan curve under conformal mapping. Duke Math. J. 40, 547-559 (1973)

[6] Carleson, L., Makarov, N.: Some results connected with Brennan's conjecture. Ark. Mat. 32, 33-62 (1994)

[7] DiBenedetto, E., Friedman, A.: The ill-posed Hele-Shaw model and the Stefan problem for supercooled water. Trans. Amer. Math. Soc. 282, 183-204 (1984)

[8] Gustafsson, B.: On a differential equation arising in a Hele-Shaw flow moving boundary problem. Ark. Mat. 22, 251-268 (1984)

[9] Hastings, M., Levitov, L.: Laplacian growth as one-dimensional turbulence. Phys. D. 116, 244-252 (1998)

[10] Hele-Shaw, H.J.S.: On the motion of a viscous fluid between two parallel plates. Nature 58, 34-36 (1898)

[11] Kesten, H.: Hitting probabilities of random walks on $\mathbb{Z}^{d}$. Stoch. Proc. Appl. 25, 165-184 (1987)

[12] Lawler, G.F.: Intersection of random walks. Birkhäuser, 1996

[13] Lawler, G.F., Schramm, O., Werner W.: Values of Brownian intersection exponents I: Halfplane exponents, II: Plane exponents. Preprints, 1999, 2000

[14] Loewner, K.: Untersuchungen über schlichte konforme Abbildungen des Einheitkreises,I. Math. Ann. 89, 103-121 (1923)

[15] Marshall, D., Rohde, S.: in preparation

[16] McMullen, C.T.: Program dla.tar.gz via http://www.harvard.edu/ ctm/.

[17] Pommerenke, Ch.: Univalent functions. Göttingen: Vandenhoeck and Ruprecht, 1975

[18] Pommerenke, Ch.: Boundary behavior of conformal maps. Berlin: Springer, 1992

[19] Saffman, P.G., Taylor, F. R.: The penetration of a fluid into a porous medium or Hele-Shaw cell containing a more viscous liquid. Proc. Royal Soc. London, Ser. A 245, 312-329 (1958)

[20] Schramm, O.: Scaling limits of loop-erased random walks and uniform spanning trees. Preprint, 1999

[21] Shraiman, B., Bensimon, D.: Singularities in nonlocal interface dynamics. Phys. Rev. A 30, 2840-2842 (1958)

[22] Vicsek, T.: Fractal growth phenomena. Singapore: World Scientific, 1989

[23] Witten, T.A., Sander, L.M.: Diffusion-limited aggregation, a kinetic phenomenon. Phys. Rev. Lett. 47, 1400-1403 (1981) 
Royal Institute of Technology, Department of Mathematics, Stockholm, S10044, SWEDEN

E-mail address: carleson@math.kth.se

California Institute of Technology, Department of Mathematics, Pasadena, CA 91125, USA

E-mail address: makarov@cco.caltech.edu 